\title{
Increased risk of endocrine autoimmunity in first-degree relatives of patients with autoimmune Addison's disease
}

\section{Marta Fichna', Piotr P Małecki', Mirela Młodzikowska', Bolesław Gębarski ${ }^{3}$, Marek Ruchała' ${ }^{1}$ and Piotr Fichna ${ }^{4}$}

${ }^{1}$ Department of Endocrinology, Metabolism and Internal Medicine, Poznań University of Medical Sciences, Poznan, Poland, ${ }^{2}$ Central Laboratory of the Poznan University of Medical Sciences Paediatric Hospital, Poznan, Poland, ${ }^{3}$ Outpatient Medical Centre Remedium, Pawlowice, Poland, and ${ }^{4}$ Department of Paediatric Diabetes and Obesity, Poznan University of Medical Sciences, Poznan, Poland
Correspondence should be addressed to M Fichna

Email

mfichna@ump.edu.pl

\begin{abstract}
Objective: Autoimmune conditions tend to cluster in subjects with Addison's disease (AD) and probably also among their relatives. The aim of the study was to estimate the frequency of the endocrine gland-specific autoantibodies in first-degree relatives of patients with AD.

Methods: Autoantibodies were investigated in 113 family members using RIA and ELISA assays. The control group comprised 143 age-matched volunteers.

Results: Autoimmune diseases were diagnosed in $38.1 \%$ relatives. Hashimoto's thyroiditis was found in 20.3\%, Graves' disease in $8.0 \%$, vitiligo and type 1 diabetes in 3.5\%, whereas AD, rheumatoid arthritis and atrophic gastritis with pernicious anaemia in $2.7 \%$ each.

All studied antibodies except for islet antigen-2 $(P=0.085)$ were significantly more frequent in AD relatives than in controls $(P<0.05)$. Antibodies to 21 -hydroxylase were detected in $6.2 \%$ relatives, thyroid peroxidase in $28.3 \%$, thyroglobulin in $19.5 \%$, glutamic acid decarboxylase in $8.0 \%$, and zinc transporter-8 in $7.1 \%$. Two and more autoantibodies were detected in $18.6 \%$ subjects. Significant gender difference was revealed only for aTPO, more common in female relatives ( $P=0.014$; OR: 3.16; $95 \% \mathrm{Cl}: 1.23-8.12)$. Circulating autoantibodies were found more frequently in the relatives of affected males $(P=0.008 ; \mathrm{OR}: 3.31 ; 95 \% \mathrm{Cl}: 1.33-8.23)$, and in family members of patients with polyendocrine autoimmunity ( $P=0.009$; OR: $3.55 ; 95 \% \mathrm{Cl}: 1.31-9.57)$.

Conclusions: This study provides evidence of increased susceptibility for the endocrine autoimmunity, especially thyroid disease, in close relatives of patients with AD. Relatives of the male AD patients and of those with autoimmune polyendocrine syndrome are at particular risk and should undergo periodic screening for autoimmune endocrine disorders.
\end{abstract}

\section{Introduction}

Primary adrenal insufficiency (Addison's disease (AD)) is a rare but life-threatening endocrine disorder, which requires lifelong glucocorticoid and mineralocorticoid replacement. Autoimmune destruction of the adrenal cortex appears the most common reason for $\mathrm{AD}$ in the
European Journal of

Endocrinology

(2020) 183, 73-81 modern countries. Patients with autoimmune adrenal failure display exceptional susceptibility for other autoimmune conditions, with coexisting disorders found in more than half individuals $(1,2,3)$. Standard combinations of the autoimmune diseases are classified 
into distinct autoimmune polyendocrine syndromes (APS) (4). Only very rare type 1 APS, which typically consists of $\mathrm{AD}$, hypoparathyroidism and/or chronic candidiasis, presents autosomal recessive inheritance of causative mutations in the AIRE gene (5). The genetics of the other, more frequent, APS forms remains less recognized. Autosomal dominant inheritance with incomplete penetrance has been proposed for APS type 2, defined as an association of $\mathrm{AD}$ with autoimmune thyroid disease (AITD) and/or type 1 diabetes (T1D), potentially associated with any other autoimmune disorder $(4,6)$. APS type 4 is sometimes further distinguished from type 2 , if $\mathrm{AD}$ is combined with autoimmune diseases other than AITD and T1D (6). Nevertheless, it seems that various autoimmune conditions tend to cluster in subjects with $\mathrm{AD}$, but also among their relatives, although precise data are lacking. Epidemiological analyses in families of patients suffering from other autoimmune disorders: Graves' disease (GD), T1D, and vitiligo confirm increased frequency of the autoimmune disorders in the relatives of the affected subjects $(7,8,9)$. To the best of our knowledge, a unique systematic analysis of the prevalence of the organ-specific serum autoantibodies in $\mathrm{AD}$ families was published half a century ago (10). However, circulating antibodies to adrenal, thyroid and gastric autoantigens were then detected by means of the complement fixation tests, which are less sensitive compared to the modern assays (10). Recently, selfreported data concerning prevalence of autoimmunity in $\mathrm{AD}$ families were collected through an international survey, although complete results have never been published (11).

Therefore, in order to provide contemporary data about the risk of autoimmunity in $\mathrm{AD}$ families, the aim of the current investigation was to estimate the frequency of the adrenal cortex, thyroid and pancreatic islet beta cell specific autoantibodies in a cohort of the first-degree relatives of patients with autoimmune AD.

\section{Patients and methods}

First-degree relatives of the subjects with AD diagnosed or followed in our tertiary endocrinology center were invited to take part in the study. Only family members of patients with confirmed autoimmune origin (positive serum autoantibodies to 21-hydroxylase) of the primary adrenocortical failure were enrolled. Relatives of individuals with other causes of adrenal insufficiency, such as congenital adrenal hyperplasia or X-linked adrenoleukodystrophy, were not recruited. Families with type 1 APS were also excluded.

The study was devised as cross-sectional medical examination with evaluation of serum autoantibodies in first-degree relatives (i.e. parents, siblings and offspring aged at least 12 years old) of patients with autoimmune AD. Medical data and serum samples were obtained from 113 first-degree relatives (45 males, 68 females) of the AD patients, including 9 parents (20.4\% of the living parents; 6 mothers and 3 fathers), 44 siblings (53.0\% of the living siblings; 29 sisters and 15 brothers), and 60 offspring (78.9\% of the living offspring; 33 daughters and 27 sons), all issued from 51 families. Their mean age was $34.8 \pm 15.4$ years.

Relatives were asked to supply their medical documentation to assess if any autoimmune disorder had already been diagnosed in the past. All participants underwent clinical examination by an endocrinologist, searching for features of the autoimmune diseases, such as goitre, vitiligo, hyperpigmentation of the skin and mucosae, or joint deformities. Ultrasonography of the thyroid was performed in all participating relatives using the Affiniti 70 sonography unit (Philips) with a $7.5 \mathrm{mHz}$ linear transducer. The ultrasound was intended to detect or confirm formerly recognized features of the AITD, especially heterogeneous, hypoechoic texture of the gland.

The control group for serologic analyses consisted of 143 consecutively consulted sex- and age-matched volunteers (mean age $35.4 \pm 12.6$ years; 54 males and 89 females) randomly recruited at the community-based family doctors' practice. Within this group two subjects appeared to be previously diagnosed with hypothyroidism, one person with psoriasis and one individual suffered from rheumatoid arthritis and vitiligo. This proportion roughly reflects the prevalence of these conditions in the population. Both relatives and controls comprised similar, small portion of smokers, 5.3 and 7.7\%, respectively.

Blood samples were taken in the morning after overnight fast, centrifuged and stored at $-20^{\circ} \mathrm{C}$ until analysed. Circulating antibodies to steroid 21-hydroxylase (a21OH), thyroid peroxidase (aTPO), thyroglobulin (aTg), glutamic acid decarboxylase (aGAD), zinc transporter- 8 (aZnT8), and tyrosine phosphatase (aIA2) were detected using RIA- and ELISA-based assays: 21-Hydroxylase Autoantibody ELISA Kit (RSR Ltd. Cardiff, UK), Anti-TPOn RIA kit, ThermoScientific (BRAHMS GmbH, Hennigsdorf, Germany), Anti-TGn RIA kit, ThermoScientific (BRAHMS GmbH, Hennigsdorf, Germany), GAD65 Ab RIA kit (DRG International Inc. Marburg, Germany), Zinc Transporter 8 Autoantibody ELISA Kit (RSR Ltd. Cardiff, UK) and IA2 Ab RIA kit (DRG International Inc. Marburg, Germany), 
respectively. RIA results were read on scintillation gamma counter Wallac 1470 Wizard (Perkin Elmer), while the absorbance from ELISA assays was analysed on ELx808 Absorbance Reader (BioTek, VT, USA) using Gen5 Microplate Reader Software.

The study protocol was approved by the Local Ethical Board at Poznan University of Medical Sciences, and all participants gave their informed consent, signed by individuals aged at least 16 years old and, in case of minors, by their legal representatives.

Statistical analysis was performed using GraphPad Prism 6.0c (GraphPad Software). Data normality was verified with Shapiro-Wilk test. Normally distributed continuous variables were compared by means of the t-Student test, while those with non-Gaussian distribution were analysed by nonparametric Mann-Whitney test. Statistical correlations were assessed by Pearson's or Spearman's rank correlation coefficient, depending on data distribution. Categorical variables were compared using $\mathrm{Chi}^{2}$ or Fisher exact test when appropriate. Associations between individual status and the presence of the autoantibodies were determined calculating the odds ratios (OR) with 95\% CIs (95\% CI). Two-tailed $P$ values of less than 0.05 were considered significant.

\section{Results}

Based upon their medical history 43 (38.1\%) of the 113 first-degree relatives of the $\mathrm{AD}$ patients had already been diagnosed with an autoimmune condition in the past, whereas $70(61.9 \%)$ of them were considered healthy at the time of the study (Table 1). AITD was the most prevalent disorder, found in $28.3 \%$ subjects: Hashimoto's thyroiditis (HT) was diagnosed in 20.3\%, while GD - in $8.0 \%$. Four (3.5\%) individuals displayed vitiligo, and another $4-\mathrm{T} 1 \mathrm{D}$. AD was formerly diagnosed in 3

Table 1 Autoimmune conditions in 113 first-degree relatives of patients with autoimmune Addison' disease.

\begin{tabular}{lcc}
\hline Disease & & Number of relatives \\
\cline { 1 - 2 } Autoimmune thyroid disease & $32(28.3 \%)$ \\
$\quad$ Hashimoto's thyroiditis & $23(20.3 \%)$ \\
$\quad$ Graves' disease & $9(8.0 \%)$ \\
Type 1 diabetes & $4(3.5 \%)$ \\
Vitiligo & $4(3.5 \%)$ \\
Addison's disease & $3(2.7 \%)$ \\
Chronic atrophic gastritis & $3(2.7 \%)$ \\
Rheumatoid arthritis & $3(2.7 \%)$ \\
Psoriasis & $2(1.8 \%)$ \\
Other & $3(2.7 \%)$ \\
\hline
\end{tabular}

siblings of the AD patients. Three persons suffered from chronic atrophic gastritis with pernicious anaemia, and 3 - from rheumatoid arthritis, while 2 others had psoriasis. Single cases of other autoimmune conditions: systemic lupus erythematosus, alopecia, and ulcerative colitis were registered. Thirty-seven relatives (32.7\%) presented unique autoimmune condition, 4 individuals (3.5\%) suffered from two disorders. Three and four coexisting autoimmune diseases were found in single subjects $(0.9 \%)$. Ultrasound thyroid examination revealed heterogeneous hypoechoic texture of the gland, typical for AITD, in 36 individuals (31.6\%).

The evaluation of antibodies against specific adrenal, thyroid and beta cell antigens demonstrated that all studied antibody specificities except for aIA2 $(P=0.085)$ were more common in $\mathrm{AD}$ relatives than in controls (Table 2). aIA2 were found in three relatives and none of the controls. Likewise, a $210 \mathrm{H}$ were not detected in any control subject, but confirmed in 7 (6.2\%) AD relatives $(P=0.003)$. aTPO were the most common autoantibodies, found in $28.3 \%$ relatives vs $6.3 \%$ controls $(P<0.001)$, followed by aTg, detected in $19.5 \%$ relatives vs $2.8 \%$ controls $(P<0.001)$. OR values indicate that a relative with $\mathrm{AD}$ is a considerable risk factor for developing $\mathrm{a} 21 \mathrm{OH}$, aTPO, aTg, aGAD or aZnT8 (Table 2). On the whole, having a first-degree relative with $\mathrm{AD}$ was associated with more than seven-fold rise in probability of developing any of the investigated antibodies (OR 7.22; 95\% CI 3.58-14.56; $P<0.001)$.

No autoantibodies were found in $68 \mathrm{AD}$ relatives (60.2\%), while 24 subjects (21.2\%) displayed positivity for just one autoantibody (Fig. 1). Two autoantibodies were detected in 12 individuals (10.6\%), three different autoantibodies in 4 subjects (3.5\%), and four autoantibodies - in $4(3.5 \%)$ persons as well. Finally, 5 autoantibodies were found in 1 first-degree relative (0.9\%). Combinations of the specific autoantibodies detected in serum of the first-degree relatives of patients with $\mathrm{AD}$ are displayed in Supplementary Table 1 (see section on supplementary materials given at the end of this article). aTPO and aTg were the most frequently coexisting antibodies, found in 12 relatives, and in 5 further individuals in association with other autoantibody specificities. Of note, most cases of positive a $21 \mathrm{OH}$ or aZnT8 appeared in conjunction with other autoimmune markers.

Gender-stratified analysis of the circulating autoantibodies in the first-degree relatives revealed significant difference only in case of aTPO, which were more common in females $(P=0.014)$, indicating more than three-fold increase of risk for aTPO-positivity 
Table 2 Comparison of the frequency of serum autoantibodies against specific adrenal, thyroid and beta cell antigens in first-degree relatives of patients with Addison's disease (AD) and healthy controls.

\begin{tabular}{l}
\hline Autoantibody \\
\hline a21OH \\
aTPO \\
aTg \\
aGAD \\
aIA2 \\
aZnT8 \\
Any autoantibody \\
\hline
\end{tabular}

\begin{tabular}{c}
\hline AD relatives $(113)$ \\
\hline $7(6.2 \%)$ \\
$32(28.3 \%)$ \\
$22(19.5 \%)$ \\
$9(8.0 \%)$ \\
$3(2.6 \%)$ \\
$8(7.1 \%)$ \\
$45(39.8 \%)$
\end{tabular}

\begin{tabular}{c}
\hline Controls $(143)$ \\
\hline 0 \\
$9(6.3 \%)$ \\
$4(2.8 \%)$ \\
$2(1.4 \%)$ \\
0 \\
$1(0.7 \%)$ \\
$12(8.4 \%)$
\end{tabular}

$\begin{array}{r}\hline \text { P value } \\ \hline \mathbf{0 . 0 0 3} \\ <0.001 \\ <0.001 \\ \mathbf{0 . 0 1 3} \\ 0.085 \\ \mathbf{0 . 0 1 2} \\ <\mathbf{0 . 0 0 1} \\ \hline\end{array}$

\begin{tabular}{c}
\hline OR \\
\hline 20.2 \\
5.88 \\
8.40 \\
6.10 \\
9.09 \\
10.9 \\
7.22 \\
\hline
\end{tabular}

\begin{tabular}{c}
\hline $\mathbf{9 5} \% \mathbf{C l}$ \\
\hline $1.14-358.0$ \\
$2.67-12.95$ \\
$2.80-25.19$ \\
$1.29-28.84$ \\
$0.46-178.0$ \\
$1.34-88.49$ \\
$3.58-14.56$ \\
\hline
\end{tabular}

a21OH, antibodies against 21-hydroxylase; aTPO, antibodies against thyroid peroxydase; aTg, antibodies against thyroglobulin; aGAD, antibodies against glutamic acid decarboxylase; alA2, antibodies against tyrosine phosphatase; aZnT8, antibodies against zinc transporter-8; OR, odds ratio.

compared to males (OR 3.16; 95\%CI 1.23-8.12) (Table 3). On the contrary, aIA2 were detected only in male relatives, although the difference did not reach statistical significance $(P=0.061)$.

An investigation of serum autoantibodies in family members with regard to their relationship with the patient was planned. However, due to low number of the enrolled parents of subjects with $\mathrm{AD}$, the comparison had to be restricted to siblings and children of patients. The analysis did not reveal significant differences in prevalence of any autoantibodies between siblings and offspring (all $P$ values $>0.05$ ) (Supplementary Table 2).

The association of the AD patient's own features with the risk of autoimmunity in the family members was also considered. The frequency of circulating autoantibodies in the relatives of affected females (32.6\%) and males $(61.5 \%)$ differed significantly $(P=0.008)$, indicating that family members of the male $\mathrm{AD}$ patients are at much higher risk for autoimmunity (OR 3.31; 95\% CI 1.33-8.23). Moreover, circulating autoantibodies were

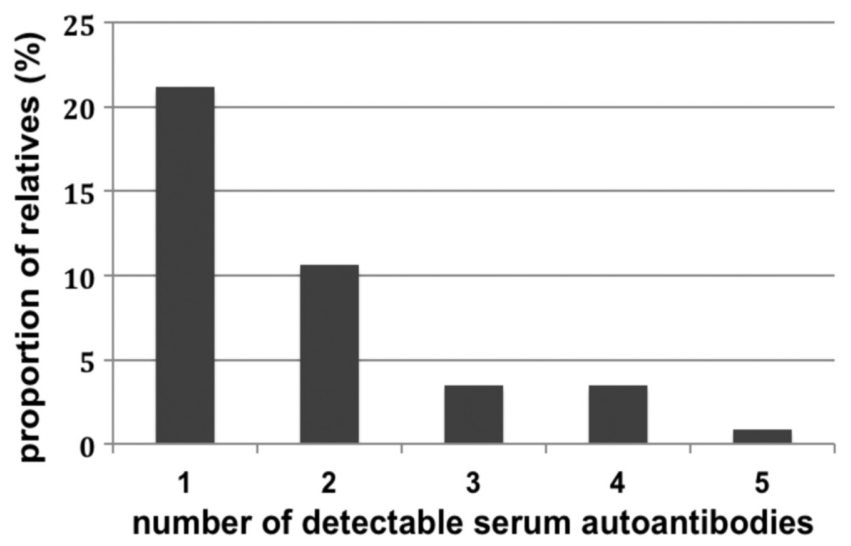

\section{Figure 1}

Proportion of the first-degree relatives of patients with autoimmune Addison's disease carrying 1-5 serum autoantibody specificities. detected in $6(20.0 \%)$ among 30 relatives of patients with isolated $\mathrm{AD}$ and 39 (47.0\%) out of 83 family members of patients with APS $(P=0.009)$. These proportions revealed more than three-fold rise in autoimmunity risk in the relatives of patients with polyendocrine autoimmunity (OR 3.55; 95\% CI 1.31-9.57). Furthermore, the number of serum autoantibodies in first-degree relatives was positively correlated with the number of the autoimmune disorders in their family members suffering from AD ( $r=0.337 ; P=0.0003)$. Of note, a comparison of the age at $\mathrm{AD}$ onset in patients from families with multiple AD cases and those without affected relatives revealed significantly younger AD manifestation in this former group $(29.5 \pm 6.5$ vs $40.2 \pm 11.5$ years, $P=0.027$ ).

\section{Discussion}

Heritable factors, which confer susceptibility toward autoimmune disease, may lead to clustering of autoimmune conditions within kinship $(12,13)$. In line,

Table 3 Comparison of the frequency of serum autoantibodies against specific adrenal, thyroid and beta cell antigens in female and male first-degree relatives of patients with Addison's disease.

\begin{tabular}{|c|c|c|c|}
\hline Autoantibody & $\begin{array}{c}\text { Female } \\
\text { relatives (68) }\end{array}$ & $\begin{array}{c}\text { Male } \\
\text { relatives (45) }\end{array}$ & P-value \\
\hline $\mathrm{a} 210 \mathrm{H}$ & $4(5.9 \%)$ & $3(6.7 \%)$ & 1.000 \\
\hline aTPO & $25(36.8 \%)$ & $7(15.5 \%)$ & 0.014 \\
\hline aTg & $13(19.1 \%)$ & $9(20.0 \%)$ & 0.908 \\
\hline aGAD & $6(8.8 \%)$ & $3(6.7 \%)$ & 1.000 \\
\hline alA2 & 0 & $3(6.7 \%)$ & 0.061 \\
\hline aZnT8 & $5(7.3 \%)$ & $3(6.7 \%)$ & 1.000 \\
\hline Any autoantibody & $30(44.1 \%)$ & $15(33.3 \%)$ & 0.569 \\
\hline
\end{tabular}

a210H, antibodies against 21-hydroxylase; aTPO, antibodies against thyroid peroxydase; aTg, antibodies against thyroglobulin; aGAD, antibodies against glutamic acid decarboxylase; alA2, antibodies against tyrosine phosphatase; aZnT8, antibodies against zinc transporter-8. 
autoimmune diseases were found in a considerable number of first-degree relatives of patients with $\mathrm{AD}$ in our study, affecting more than $38 \%$ of the family members. This proportion largely outnumbers autoimmunity estimates, which oscillate between 3.2 and $9.4 \%$ of the general population, depending on the examined population and the number of the considered diseases $(14,15)$. AITD was the most common condition diagnosed in relatives of the AD patients, found in $28.3 \%$ family members. Data from the medical records were largely confirmed by the results of the thyroid ultrasound - images characteristic for AITD were detected in 31.6\%. Epidemiological Polish data are lacking, but estimates from other Caucasian populations from the iodine-replete regions indicate the prevalence of HT at approximate level of 5\% in females and $<2 \%$ in males $(14,16,17)$. Hyperthyroidism is diagnosed in 0.3-2.0\% Caucasian Europeans and GD accounts for approximately two-thirds of cases (17). Data from German families with AITD demonstrate clustering of the disease in children and siblings of the affected subjects, with over 20-fold increase of the risk for HT and 7-fold that for GD (18). Likewise, $\mathrm{AD}$ in a relative seems to be risk factor for AITD in the family members.

Other relatively common autoimmune disorders in the studied cohort comprised T1D and vitiligo, each found in $3.5 \%$ of the $\mathrm{AD}$ relatives. T1D prevalence in general population is ethnicity dependent, and in Poland estimated for nearly $0.5 \%$ (19). Worldwide prevalence of vitiligo ranges between $0.4 \%$ and $2.0 \%$ (20). Finally, $\mathrm{AD}$, rheumatoid arthritis and chronic atrophic gastritis with pernicious anaemia were each diagnosed in $2.7 \%$ relatives, whereas in general population they are found in $<1 \%(21,22)$. Data from the Swedish and Norwegian $\mathrm{AD}$ registries corroborate familial aggregation of the adrenal failure, which affects $6.4-9.7 \%$ of the family members $(1,23)$. Six $(5.3 \%)$ of the currently studied relatives suffered from more than one autoimmune disease, providing evidence of the remarkable familial predilection for autoimmunity. Moreover, some participants admitted that there had been suspicion of an autoimmune disorder, which has not been further investigated. Therefore, our results, based solely upon medically proven cases with supporting documentation, might still underestimate the clustering of autoimmune conditions in the $\mathrm{AD}$ families. An international mail survey conducted among patients with AD revealed increased prevalence of $\mathrm{AD}$, thyroid conditions, $\mathrm{T} 1 \mathrm{D}$, vitamin B12 deficiency and vitiligo in their extended families compared to gender- and age-matched controls (11). However, the study was based upon self-reported autoimmune diseases in family members without access to their medical documentation.

In order to obtain unbiased data, our analysis was specifically focused on detection of the circulating autoantibodies in first-degree relatives of patients with autoimmune AD. Autoimmune endocrine disorders usually develop over several months or even years and circulating antibodies against specific autoantigens may herald clinical onset of T1D, AITD, and AD in the predisposed populations $(24,25,26,27,28,29)$. Our investigation revealed that all studied autoantibodies with the exception of aIA2 appeared significantly more frequently in the relatives of the $\mathrm{AD}$ patients compared to the control population. However, aIA2 were only detected in three males, a number not sufficient to obtain statistical significance.

Antibodies against 21-hydroxylase are highly specific for autoimmune AD. They are found in $72-87 \%$ of the affected individuals, while their prevalence in general population remains $<1 \%(1,3,30,31,32)$ but rises up to $1.4-1.7 \%$ in T1D or $\operatorname{AITD}(33,34)$. a21OH are valuable markers of incipient $\mathrm{AD}$, especially in children, even though evolution to clinical adrenal failure may take several years $(27,28,29,33,34)$. Some data support that although class II HLA alleles increase susceptibility for $\mathrm{AD}$, class I alleles, namely HLA-B15, may protect a21OHpositive subjects from progression to clinical disease, hence affect the course of autoimmune disorder $(35,36)$. On the contrary, homozygosity of the MHC class I (MIC)-related gene, MICA5.1 polymorphism allows to identify likely progressors between the high-risk DR3/4-DQB1*0302 a21OH-positive subjects (37). In the currently studied cohort, 3 out of 7 a21OH-positive individuals have already been diagnosed with adrenal insufficiency, whereas 4 subjects remained asymptomatic. Previous investigation of serum autoantibodies in first-degree relatives revealed increased prevalence of the adrenal antibodies in parents (11.1 vs $4.2 \%$ ) and siblings (3.0 vs 1.4\%) of patients with APS type 2 compared to the control subjects, but the differences did not reach statistical significance (10). However, that study, which relied on determination of antibodies against the microsomes of adrenocortical cells, was only able to detect autoantibodies in $58.6 \%$ patients with AD.

Circulating anti-thyroid antibodies, aTPO and aTg, were the most frequent finding in the relatives of the AD patients in our study, and their combined incidence was also common. These markers are detectable both in HT (aTPO in over 90\% and aTg in approximately 70\%) and GD (aTPO in up to 60\% and aTg in 40\%, respectively) 
(38). Their frequencies in iodine-sufficient populations range between 5 and $18 \%$ and tend to rise in elderly women (17). Serum aTPO and aTg are relatively common in subjects suffering from other endocrine conditions, such as $\mathrm{T} 1 \mathrm{D}$ and $\mathrm{AD}$, and in systemic autoimmunity $(2,3$, $39,40)$. A prospective study in close relatives of patients with T1D revealed that by 20 years of age the overall risk of developing aTPO exceeds 10\% (40). In our analysis, aTPO were the only autoantibodies, which displayed gender difference, with more than twice increased preponderance in females. This observation remains in line with considerably higher prevalence of AITD among females across all populations $(16,17,18)$.

On the contrary, no gender-related differences were detected in our analysis of aGAD and aZnT8, both specific for pancreatic beta cell autoimmunity. Nevertheless, these autoantibodies were significantly more frequent among the $\mathrm{AD}$ relatives ( 8.0 and $7.1 \%$, respectively) compared to controls (1.4 and $0.7 \%$, respectively). aGAD are found in $67-84 \%$ subjects with newly diagnosed T1D and in 1-4\% of the general population $(41,42,43)$. Notably, aGAD are detected in $54-63 \%$ siblings of children with T1D, and their high titers indicate increased risk for disease progression $(44,45)$. Studies in the $\mathrm{AD}$ cohorts from Poland, Norway and Italy confirm about $20 \%$ prevalence of aGAD, and consequently, elevated risk for T1D (1, 3, 46). Although less frequent than aGAD, aZnT8 seem quite common in $\mathrm{AD}$ patients, found in $8.5 \%$ subjects (46). These autoantibodies are detectable in $60-80 \%$ of newly diagnosed T1D compared to $<2 \%$ healthy controls (47). Furthermore, aZnT8 are useful predictive tool in firstdegree relatives of the T1D patients $(48,49)$. Altogether, increased prevalence of beta cell-specific autoantibodies found in close relatives of patients suffering from $A D$ corroborates considerable risk for T1D among those subjects. Of note, aZnT8 in our study were usually identified in combination with other autoantibodies. Detection of aZnT8 in autoimmune adult-onset diabetes associates with the presence and higher titers of aTPO hence may be a sign of the risk for concomitant AITD (43). Therefore, positive aZnT8 could indicate increased threat for polyglandular autoimmunity. Indeed, two and more autoantibodies were detected in nearly one fifth (18.6\%) of family members of the AD patients in our cohort. Multiple autoantibody positivity provides further evidence of exceptional propensity to autoimmune reactions among the relatives of individuals suffering from $\mathrm{AD}$.

An intriguing novel finding from our analysis relies upon substantially elevated prevalence of serum autoantibodies among the relatives of the males with $\mathrm{AD}$, and those suffering from multi-organ autoimmunity. Although $\mathrm{AD}$, as the majority of the autoimmune conditions, is more prevalent in females, male patients present significantly earlier disease onset $(2,3,50$, 51). Moreover, observations of the preferential male transmission of disease to their offspring were reported for T1D (52). These traits could suggest more prominent heritable impact on disease development in males. As a proof, monogenic disorders such as APS type 1, usually develop much earlier during lifetime than complex diseases. In line, earlier $\mathrm{AD}$ manifestation in patients with familial cases of $\mathrm{AD}$, compared to those with no affected relatives, further supports considerable role of the genetic factors. However, mean age at disease onset in patients with isolated $\mathrm{AD}(28.7 \pm 12.2$ years $)$ was also significantly lower compared to patients with APS type 2 or $4(37.4 \pm 12.1$ years, $P=0.008)$, who are expected to bear particular genetic susceptibility to autoimmune conditions. Nonetheless, AD is not necessarily the first clinical manifestation of APS, which may start by any of its components, thus this finding needs further evaluation in separate cohorts. Anyway, polyglandular autoimmunity is probably due to a mixture of genetically mediated, minor immune dysfunctions, which combined with some yet unrecognised environmental triggers contribute to the altered immune regulation. Notably, the number of serum autoantibodies found in the first-degree relatives was positively correlated with the number of the autoimmune disorders in their family members with $\mathrm{AD}$, a feature that reflects strong genetic liability for the misguided immune reactions. Many genetic factors which increase susceptibility to non-type 1 APS autoimmune AD are shared by several autoimmune conditions (53). For instance class II HLA alleles and polymorphic variants of PTPN22, CTLA-4, and $B A C H 2$ genes are associated with $\mathrm{AD}, \mathrm{AITD}$ and T1D $(54,55)$. Although predisposing alleles are rather rare in the population, the estimated heritability of $\mathrm{AD}$ equal to 0.97 (95\% CI 0.88-0.99) suggests considerable genetic influence (56). To the best of our knowledge, no data with regard to heritability of APS type 2 were published. However, most of the genetic predisposition for polyendocrine autoimmunity can be attributed to class II HLA alleles, which are the same as those predisposing for T1D $(57,58)$. Polymorphisms of the PTPN22 and CTLA-4 genes were proposed as prediction tools of polyglandular autoimmunity in patients with single autoimmune endocrine disorders and relatives of subjects suffering from APS (59). 
The results of the current investigation indicate the need for further studies of the immunogenetic pathomechanism for the endocrine autoimmunity. The major weakness of our analysis consists of limited number of the studied relatives. In particular, the number of the enrolled parents was too low to allow comparison with other family members. Mean age of the AD patients, whose relatives we studied, was $52.7 \pm 13.9$ years (range 24-78) hence their parents were respectively older. At the time of the study many of these parents were not alive, lived at a long distance or were unwilling to participate due other diseases and disabilities. Definitely, a larger cohort of close relatives would enable to perform powerful molecular analyses of the disease predisposing polymorphisms and correlate serologic findings with the genetic makeup. However, the strength of our study relies on well-documented individual data from each participant, excluding risk of any misclassification. Unfortunately, epidemiological statistics concerning the prevalence of autoimmune endocrine diseases in Poland are not available, due to lack of the nationwide registries. Hence we had to compare the frequency of serum autoantibodies with a surrogate control cohort consisting of the patients of the family doctors practice. Nevertheless, this study gives a valuable insight into the burden of autoimmunity in the families of patients suffering from AD.

In conclusion, the current study is the unique modern analysis of serum autoantibodies in the immediate relatives of patients with autoimmune AD. Our findings provide evidence of increased risk for the endocrine autoimmunity, especially AITD, in this group. Relatives of the male AD patients and of those with APS rather than isolated $\mathrm{AD}$ are at particular risk of developing organspecific autoantibodies, and should undergo periodic screening for the autoimmune endocrine disorders. Efficient detection of preclinical stages of disorders such as AAD or T1D may prevent life-threatening crisis at disease onset and in future will probably allow undertaking some preventive measures before the development of clinical symptoms.

\section{Supplementary materials}

This is linked to the online version of the paper at https://doi.org/10.1530/ EJE-20-0150.

\section{Declaration of interest}

The authors declare that there is no conflict of interest that could be perceived as prejudicing the impartiality of this study.

\section{Funding}

This research did not receive any specific grant from any funding agency in the public, commercial or not-for-profit sector.

\section{Acknowledgements}

The authors would like to warmly thank all our patients with Addison's disease, their relatives and healthy volunteers for their participation and comprehension.

\section{References}

1 Erichsen MM, Lovas K, Skinningsrud B, Wolff AB, Undlien DE, Svartberg J, Fougner KJ, Berg TJ, Bolleslev J, Mella B et al. Clinical, immunological, and genetic features of autoimmune primary adrenal insufficiency: observations from a Norwegian registry. Journal of Clinical Endocrinology and Metabolism 200994 4882-4890. (https:// doi.org/10.1210/jc.2009-1368)

2 Fichna M, Fichna P, Gryczynska M, Walkowiak J, Zurawek M \& Sowiski J. Screening for associated autoimmune disorders in Polish patients with Addison's disease. Endocrine 201037 349-360. (https:// doi.org/10.1007/s12020-010-9312-x)

3 Betterle C, Scarpa R, Garelli S, Morlin L, Lazzarotto F, Presotto F, Coco G, Masiero S, Parolo A, Albergoni MP et al. Addison's disease: a survey on 633 patients in Padova. European Journal of Endocrinology 2013169 773-784. (https://doi.org/10.1530/EJE-13-0528)

4 Neufeld M \& Blizzard RM. Polyglandular autoimmune diseases. In Symposium on Autoimmune Aspects of Endocrine Disorders, pp. 357-365. Eds A Pinchera, D Doniach, GF Fenzi \& L Baschieri. New York: Academic Press, 1980.

5 Orlova EM, Sozaeva LS, Kareva MA, Oftedal BE, Wolff ASB, Breivik L, Zakharova EY, Ivanova ON, Kampe O, Dedov II et al. Expanding the phenotypic and genotypic landscape of autoimmune polyendocrine syndrome Type 1. Journal of Clinical Endocrinology and Metabolism 2017102 3546-3556. (https://doi.org/10.1210/jc.2017-00139)

6 Betterle C, Dal Pra C, Mantero F \& Zanchetta R. Autoimmune adrenal insufficiency and autoimmune polyendocrine syndromes: autoantibodies, autoantigens, and their applicability in diagnosis and disease prediction. Endocrine Reviews 200223 327-364. (https://doi. org/10.1210/edrv.23.3.0466)

7 Hemminki K, Li X, Sundquist J \& Sundquist K. The epidemiology of Graves' disease: evidence of a genetic and an environmental contribution. Journal of Autoimmunity 201034 J307-J313. (https:// doi.org/10.1016/j.jaut.2009.11.019)

8 Hemminki K, Li X, Sundquist J \& Sundquist K. Familial association between type 1 diabetes and other autoimmune and related diseases. Diabetologia 200952 1820-1828. (https://doi.org/10.1007/s00125009-1427-3)

9 Narita T, Oiso N, Fukai K, Kabashima K, Kawada A \& Suzuki T. Generalized vitiligo and associated autoimmune diseases in Japanese patients and their families. Allergology International 201160 505-508. (https://doi.org/10.2332/allergolint.11-OA-0303)

10 Spinner MW, Blizzard RM, Gibbs J, Abbey H \& Childs B. Familial distributions of organ specific antibodies in the blood of patients with Addison's disease and hypoparathyroidism and their relatives. Clinical and Experimental Immunology 19695 461-468.

11 White K, Wass J \& Elliott A. Inheritance in autoimmune Addison's: the extended family profile. Endocrine Abstracts 200713 P114.

12 Tromer Y, Dolan LM, Kahaly G, Divers J, D'Agostino Jr RB, Imperatore G, Dabelea D, Marcovina S, Back MH, Pihoker C et al. Genome wide identification of new genes and pathways in patients with both autoimmune thyroiditis and type 1 diabetes. Journal of Autoimmunity 201560 32-39. (https://doi.org/10.1016/j. jaut.2015.03.006) 
13 Marquez A, Kerick M, Zhernakova A, Gutierrez-Achury J, Chen WM, Onengut-Gumuscu S, Gonzalez-Alvaro I, Rodriguez-Rodriguez L, Rios-Fernandez R, Gonzalez-Gay MA et al. Meta-analysis of Immunochip data of four autoimmune diseases reveals novel singledisease and cross-phenotype associations. Genome Medicine 201810 97. (https://doi.org/10.1186/s13073-018-0604-8)

14 Jacobson DL, Gange SJ, Rose NR \& Graham NM. Epidemiology and estimated population burden of selected autoimmune diseases in the United States. Clinical Immunology and Immunopathology 199784 223-243. (https://doi.org/10.1006/clin.1997.4412)

15 Cooper GS, Bynum ML \& Somers EC. Recent insights in the epidemiology of autoimmune diseases: improved prevalence estimates and understanding of clustering of diseases. Journal of Autoimmunity 200933 197-207. (https://doi.org/10.1016/j. jaut.2009.09.008)

16 McLeod DS \& Cooper DS. The incidence and prevalence of thyroid autoimmunity. Endocrine 201242 252-265. (https://doi.org/10.1007/ s12020-012-9703-2)

17 Vanderpump MPJ, Michael W \& Tunbridge G. The epidemiology of autoimmune thyroid disease. In Autoimmune Endocrinopathies, pp. 141-162. Ed R Volpé. Totowa NJ: Humana Press, 1999.

18 Dittmar M, Libich C, Brenzel T \& Kahaly GJ. Increased familial clustering of autoimmune thyroid diseases. Hormone and Metabolic Research 201143 200-204. (https://doi. org/10.1055/s-0031-1271619)

19 Walicka M, Chlebus M, Brzozowska M, Śliwczyński A, Jędrzejczyk T, Kania L, Puzianowska-Kuźnicka M \& Franek E. Prevalence of diabetes in Poland in the years 2010-2014. Clinical Diabetology 20154 232-237. (https://doi.org/10.5603/DK.2015.0031)

20 Silverberg NB. The epidemiology of vitiligo. Current Dermatology Reports 20154 36-43. (https://doi.org/10.1007/s13671-014-0098-6)

21 Batko B, Stajszyk M, Swierkot J, Urbanski K, Raciborski F, Jedrzejewski M \& Wiland P. Prevalence and clinical characteristics of rheumatoid arthritis in Poland: a nationwide study. Archives of Medical Science 201915 134-140. (https://doi.org/10.5114/ aoms.2017.71371)

22 Meyer G, Neumann K, Badenhoop K \& Linder R. Increasing prevalence of Addison's disease in German females: health insurance data 2008-2012. European Journal of Endocrinology 2014170 367-373. (https://doi.org/10.1530/EJE-13-0756)

23 Dalin F, Nordling Eriksson G, Dahlqvist P, Hallgren Å, Wahlberg J, Okwall O, Soderberg S, Ronnelid J, Olcen P, Winqvist $\mathrm{O}$ et al. Clinical and immunological characteristics of autoimmune Addison disease: a nationwide Swedish multicenter study. Journal of Clinical Endocrinology and Metabolism 2017102 379-389. (https://doi. org/10.1210/jc.2016-2522)

24 Kimpimaki T, Kulmala P, Savola K, Vahasalo P, Reijonen H, Ilonen J, Akerblom HK \& Knip M. Disease-associated autoantibodies as surrogate markers of type 1 diabetes in young children at increased genetic risk. Childhood Diabetes in Finland Study Group. Journal of Clinical Endocrinology and Metabolism 200085 1126-1132. (https:// doi.org/10.1210/jcem.85.3.6466)

25 De Grijse J, Asanghanwa M, Nouthe B, Albrecher N, Goubert P, Vermaulen I, Van Der Meeren S, Decochez K, Weets I, Keymeulen B et al. Predictive power of screening for antibodies against insulinomaassociated protein 2 beta (IA-2beta) and zinc transporter- 8 to select first-degree relatives of type 1 diabetic patients with risk of rapid progression to clinical onset of the disease: implications for prevention trials. Diabetologia 201053 517-524. (https://doi. org/10.1007/s00125-009-1618-y)

26 Jonsdottir B, Larsson C, Carlsson A, Forsander G, Ivarsson SA, Lernmark Å, Ludvigsson J, Marcus C, Samuelsson U, Ortqvist E et al. Thyroid and islet autoantibodies predict autoimmune thyroid disease at Type 1 diabetes diagnosis. Journal of Clinical Endocrinology and Metabolism 2017102 1277-1285. (https://doi.org/10.1210/jc.20162335)
27 Betterle C, Volpato M, Rees Smith B, Furmaniak J, Chen S, Zanchetta R, Greggio NA, Pedini B, Boscaro M \& Presotto II F. Adrenal cortex and steroid 21-hydroxylase autoantibodies in children with organ-specific autoimmune diseases: markers of high progression to clinical Addison's disease. Journal of Clinical Endocrinology and Metabolism 199782 939-942. (https://doi. org/10.1210/jcem.82.3.3849)

28 Coco G, Dal Pra C, Presotto F, Albergoni MP, Canova C, Pedini B, Zanchetta R, Chen S, Furmaniak J, Rees Smith B et al. Estimated risk for developing autoimmune Addison's disease in patients with adrenal cortex autoantibodies. Journal of Clinical Endocrinology and Metabolism 200691 1637-1645. (https://doi.org/10.1210/jc.20050860)

29 Naletto L, Frigo A, Ceccato F, Sabbadin C, Scarpa R, Presotto F, Dalla Costa M, Faggian D, Plebani M, Censi S et al. The natural history of autoimmune Addison's disease from the detection of autoantibodies to development of the disease: a long follow-up study on 143 patients. European Journal of Endocrinology 2019180 223-234. (https://doi.org/10.1530/EJE-18-0313)

30 Bednarek J, Furmaniak J, Wedlock N, Kiso Y, BaumannAntczak A, Fowler S, Krishnan H, Craft JA \& Rees Smith B. Steroid 21-hydroxylase is a major autoantigen involved in adult onset autoimmune Addison's disease. FEBS Letters 1992309 51-55. (https://doi.org/10.1016/0014-5793(92)80737-2)

31 Colls J, Betterle C, Volpato M, Prentice L, Smith BR \& Furmaniak J. Immunoprecipitation assay for autoantibodies to steroid 21-hydroxylase in autoimmune adrenal diseases. Clinical Chemistry 199541 375-380. (https://doi.org/10.1093/clinchem/41.3.375)

32 Betterle C, Coco G \& Zanchetta R. Adrenal cortex autoantibodies in subjects with normal adrenal function. Best Practice and Research: Clinical Endocrinology and Metabolism 200519 85-99. (https://doi. org/10.1016/j.beem.2004.11.008)

33 Falorni A, Laureti S, Nikoshokov A, Picchio ML, Hallengren B, Vandewalle CL, Gorus FK, Tortioli C, Luthman H, Brunetti P et al. 21-Hydroxylase autoantibodies in adult patients with endocrine autoimmune diseases are highly specific for Addison's disease. Belgian Diabetes Registry. Clinical and Experimental Immunology 1997107 341-346. (https://doi.org/10.1111/j.1365-2249.1997.262ce1153.x)

34 Barker JM, Ide A, Hostetler C, Yu L, Miao D, Fain PR, Eisenbarth GS $\&$ Gottlieb PA. Endocrine and immunogenetic testing in individuals with type 1 diabetes and 21-hydroxylase autoantibodies: Addison's disease in a high-risk population. Journal of Clinical Endocrinology and Metabolism 200590 128-134. (https://doi.org/10.1210/jc.20040874)

35 Baker PR, Baschal EE, Fain PR, Nanduri P, Triolo TM, Siebert JC, Armstrong TK, Babu SR, Rewers MJ, Gottlieb PA et al. Dominant suppression of Addison's disease associated with HLA-B15. Journal of Clinical Endocrinology and Metabolism 201196 2154-2162. (https:// doi.org/10.1210/jc.2010-2964)

36 Baker P, Fain P, Kahles H, Yu L, Hutton J, Wenzlau J, Rewers M, Badenhoop K \& Eisenbarth G. Genetic determinants of 21-hydroxylase autoantibodies amongst patients of the Type 1 Diabetes Genetics Consortium. Journal of Clinical Endocrinology and Metabolism 201297 E1573-E1578. (https://doi.org/10.1210/jc.20112824)

37 Triolo TM, Baschal EE, Armstrong TK, Toews CS, Fain PR, Rewers MJ, Yu L, Miao D, Eisenbarth GS, Gottlieb PA et al. Homozygosity of the polymorphism MICA5.1 identifies extreme risk of progression to overt adrenal insufficiency among 21-hydroxylase antibody-positive patients with type 1 diabetes. Journal of Clinical Endocrinology and Metabolism 200994 4517-4523. (https://doi.org/10.1210/jc.20091308)

38 Ragusa F, Fallahi P, Elia G, Gonnella D, Paparo SR, Guysti C, Churilov LP, Ferrari SM \& Antonelli A. Hashimotos' thyroiditis: epidemiology, pathogenesis, clinic and therapy. Best Practice and 
Research in Clinical Endocrinology and Metabolism 201926101367. (https://doi.org/10.1016/j.beem.2019.101367)

39 Lazurova I \& Benhatchi K. Autoimmune thyroid diseases and nonorganspecific autoimmunity. Polskie Archiwum Medycyny Wewnetrznej 2012122 (Supplement 1) 55-59. (https://doi. org/10.20452/pamw.1499)

40 Winkler C, Jolink M, Knopff A, Kwarteng NA, Achenbach P, Bonifacio E \& Ziegler AG. Age, HLA, and sex define a marked risk of organ-specific autoimmunity in first-degree relatives of patients with type 1 diabetes. Diabetes Care 201942 1684-1691. (https://doi. org/10.2337/dc19-0315)

41 Hagopian WA, Sanjeevi CB, Kockum I, Landin-Olsson M, Karlsen AE, Sundkvist G, Dahlquist G, Palmer J \& Lernmark A. Glutamate decarboxylase-, insulin-, and islet cell-antibodies and HLA typing to detect diabetes in a general population-based study of Swedish children. Journal of Clinical Investigation 199595 1505-1511. (https:// doi.org/10.1172/JCI117822)

42 Betterle C, Lazzarotto F, Fusari A, Zanchetta R, Bendini S, Pedini B, Moscon A \& Presotto F. Pancreatic autoantibodies in Italian patients with newly diagnosed type 1 diabetes mellitus over the age of 20 years. Acta Diabetologica 200643 79-83. (https://doi.org/10.1007/ s00592-006-0217-3)

43 Rogowicz-Frontczak A, Zozulinska-Ziolkiewicz D, Litwinowicz M, Niedziwiecki P, Wyka K \& Wierusz-Wysocka B. Are zinc transporter type 8 antibodies a marker of autoimmune thyroiditis in non-obese adults with new-onset diabetes? European Journal of Endocrinology 2014170 651-658. (https://doi.org/10.1530/EJE-13-0901)

44 Karagzuel G, Simsek S, Deger O \& Okten A. Screening of diabetes, thyroid, and celiac diseases-related autoantibodies in a sample of Turkish children with type 1 diabetes and their siblings. Diabetes Research and Clinical Practice 200880 238-243. (https://doi. org/10.1016/j.diabres.2007.12.007)

45 Bingley PJ, Boulware DC, Krischer JP \& Type 1 Diabetes TrialNet Study Group. The implications of autoantibodies to a single islet antygen in relatives with normal glucose tolerance: development of other autoantibodies and progression to type 1 diabetes. Diabetologia 201659 542-549. (https://doi.org/10.1007/s00125-015-3830-2)

46 Fichna M, Rogowicz-Frontczak A, Zurawek M, Fichna P, Gryczynska M, Zozulinska-Ziolkiewicz D \& Ruchala M. Positive autoantibodies to ZnT8 indicate elevated risk for additional autoimmune conditions in patients with Addison's disease. Endocrine 201653 249-257. (https://doi.org/10.1007/s12020-016-0916-7)

47 Wenzlau JM, Juhl K, Yu L, Moua O, Sarkar SA, Gottlieb P, Rewers M, Eisenbarth GS, Jensen J, Davidson HW et al. The cation efflux transporter ZnT8 (Slc30A8) is a major autoantigen in human type 1 diabetes. PNAS 2007104 17040-17045. (https://doi.org/10.1073/ pnas.0705894104)

48 Gorus FK, Balti EV, Vermeulen I, Demeester S, Van Dalem A, Costa O, Dorchy H, Tenoutasse S, Mouraux T, De Block C et al. Screening for insulinoma antigen 2 and zinc transporter 8 autoantibodies: a costeffective and age-independent strategy to identify rapid progressors to clinical onset among relatives of type 1 diabetic patients. Clinical and Experimental Immunology 2013171 82-90. (https://doi. org/10.1111/j.1365-2249.2012.04675.x)

49 Incani M, Serafini C, Satta C, Perra L, Scano F, Frongia P, Ricciardi R, Ripoli C, Soro M, Strazzera A et al. High prevalence of diabetesspecific autoimmunity in first-degree relatives of Sardinian patients with type 1 diabetes. Diabetes/Metabolism Research and Reviews 2017 33 e2864. (https://doi.org/10.1002/dmrr.2864)

50 Irvine WJ, Stewart AG \& Scarth L. A clinical and immunological study of adrenocortical insufficiency (Addison's disease). Clinical and Experimental Immunology 19672 31-70.

51 Ji J, Sundquist J \& Sundquist K. Gender-specific incidence of autoimmune diseases from national registers. Journal of Autoimmunity 201669 102-106. (https://doi.org/10.1016/j.jaut.2016.03.003)

52 Harjutsalo V, Reunanen A \& Tuomilehto J. Differential transmission of type 1 diabetes from diabetic fathers and mothers to their offspring. Diabetes 200655 1517-1524. (https://doi.org/10.2337/ db05-1296)

53 Zhernakova A, Withoff S \& Wijmenga C. Clinical implications of shared genetics and pathogenesis in autoimmune diseases. Nature Reviews: Endocrinology 20139 646-659. (https://doi.org/10.1038/ nrendo.2013.161)

54 Mitchell AL \& Pearce SH. Autoimmune Addison's disease: pathophysiology and genetic complexity. Nature Reviews in Endocrinology 20128 306-316. (https://doi.org/10.1038/ nrendo.2011.245)

55 Pazderska A, Oftedal BE, Napier CM, Ainsworth HF, Husebye ES, Cordell HJ, Pearce SH \& Michell AL. A variant in the BACH2 gene is associated with susceptibility to autoimmune Addison's disease in humans. Journal of Clinical Endocrinology and Metabolism 2016101 3865-3869. (https://doi.org/10.1210/jc.2016-2368)

56 Skov J, Hoijer J, Magnusson PKE, Ludvigsson JF, Kampe O \& Bensing S. Heritability of Addison's disease and prevalence of associated autoimmunity in a cohort of 112,100 Swedish twins. Endocrine 201758 521-527. (https://doi.org/10.1007/s12020-0171441-z)

57 Weinstock C, Matheis N, Barkia S, Haager MC, Janson A, Markovic A, Bux J \& Kahaly GJ. Autoimmune polyglandular syndrome type 2 shows the same HLA class II pattern as type 1 diabetes. Tissue Antigens 201177 317-324. (https://doi.org/10.1111/j.13990039.2011.01634.x)

58 Flesch BK, Matheis N, Alt T, Weinstock C, Bux J \& Kahaly GJ. HLA class II haplotypes differentiate between the adult autoimmune polyglandular syndrome types II and III. Journal of Clinical Endocrinology and Metabolism 201499 E177-E182. (https://doi. org/10.1210/jc.2013-2852)

59 Houcken J, Degenhart C, Bender K, Konig J, Frommer L \& Kahaly GJ. PTPN22 and CTLA-4 polymorphisms are associated with polyglandular autoimmunity. Journal of Clinical Endocrinology and Metabolism 2018103 1977-1984. (https://doi.org/10.1210/jc.201702577)

Received 23 February 2020

Revised version received 19 April 2020

Accepted 29 April 2020 